\title{
Stage IVA Cervical Cancer FIGO 2018
}

National Cancer Institute

\section{Source}

National Cancer Institute. Stage IVA Cervical Cancer FIGO 2018. NCI Thesaurus. Code C162246.

The cervical carcinoma has spread to adjacent pelvic organs. (FIGO 2018) 